\title{
The Use of Native Halophytes in Landscape Design in The Central Anatolia, Turkey
}

\author{
Coşkun Sağlam ${ }^{*}$, Serpil Önder² \\ ${ }^{1}$ Çumra School of Applied Science, Selçuk University, 42500 Çumra/Konya, Turkey \\ ${ }^{2}$ Department of Landscape Architecture, Faculty of Agriculture, Selçuk University, 42075 Selçuklu/Konya, Turkey
}

\section{A R T I C LE INFO}

\section{Research Articles}

Received 23 April 2018

Accepted 25 October 2018

Keywords:

Landscape values

Natural halophytes

Central Anatolia

Revegetation

Sustainability

${ }^{*}$ Corresponding Author:

E-mail: csaglam@selcuk.edu.tr

\section{A B S T R A C T}

In this study, the usability of some herbaceous halophytes grown naturally in salt marshes that dry most of the year in Central Anatolia Region were investigated in landscape design. Within the scope of the research, in the years of 2016 and 2017, seasonal field studies were carried out in saline habitats in the vicinity of Konya, Ankara, Aksaray and Nevşehir, and taken photographs and herbarium samples of halophytic plant species. The general botanical and ecological characteristics of the selected species are given and the values used in landscape design have been determined considering the aesthetic and functional properties. As a result of the field studies carried out during the vegetation periods, 59 halophytic plant species, belonging to 38 genera and 19 families that could be used in landscape design were identified. The most representative family was the Asteraceae with 11 species, followed by Plumbaginaceae $(9$ species $)$ and Chenopodiaceae ( 8 species). The genus Limonium from Plumbaginaceae family is well represented with 8 species for landscape use. The endemism rate of halophytes used in landscape design is $42 \%$ ( 25 species) in the research area. The most common uses in landscape design are determined in roof gardens by 49 species, followed in ground conservation and erosion prevention by 31 species. Since these halophytic species, which are mostly succulent and endemic, are well adapted into both wet and dry areas. Their use in landscape design is of great importance for restoration of arid and barren land, which may increase as a result of global climate change, conservation of biodiversity as well as sustainable agricultural practices.

Türk Tarım - Gıda Bilim ve Teknoloji Dergisi, 6(12): 1718-1726, 2018

\section{İç Anadolu'da (Türkiye) Doğal Halofitlerin Peyzaj Tasarımında Kullanılması}

\section{A K A L E B İ L G İ S İ}

\section{Araştırma Makalesi}

Geliş 23 Nisan 2018

Kabul 25 Ekim 2018

Anahtar Kelimeler:

Peyzaj değerleri

Doğal halofitler

İç Anadolu

Yeniden bitkilendirme

Sürdürülebilirlik

\begin{tabular}{l}
${ }^{\text {NSorumlu Yazar: }}$ \\
\hline E-mail: csaglam@ selcuk.edu.tr
\end{tabular}

\section{Ö Z}

Bu çalışmada, İç Anadolu bölgesinde yılın büyük bölümünde kuruyan tuzlu bataklıklarda doğal olarak yetişen bazı otsu halofitlerin peyzaj tasarımında kullanılabilirlikleri araştırılmıştır. Araştırma kapsamında 2016 ve 2017 yıllarında Konya, Ankara, Aksaray ve Nevşehir civarında bulunan tuzlu habitatlarda vejetasyon döneminde periyodik olarak alan çalışmaları yapılmış ve halofit bitki türlerine ait fotoğraflar ve herbaryum örnekleri alınmıştır. Seçilen türlerin genel botanik ve ekolojik özellikleri verilmiş, estetik ve fonksiyonel özellikleri dikkate alınarak peyzaj tasarımında kullanım değerleri belirlenmiştir. Araştırma alanında bulunan tuzlu habitatlarda vejetasyon dönemlerinde yapılan saha çalışmaları sonucunda peyzaj tasarımında kullanılabilecek 19 familya ve 38 cinse ait 59 halofit bitki türü tespit edilmiştir. Bu türlerden 25 tanesi Türkiye için endemik olup endemizm oran1 \%42'dir. 11 tür ile Asteraceae en fazla temsil edilen familya olurken, ardından 9 tür ile Plumbaginaceae ve 8 tür ile Chenopodiaceae familyası izlemiştir. Plumbaginaceae familyasından Limonium cinsi, 8 tür ile peyzaj tasarımında en fazla kullanım potansiyeli olan cins olmuştur. Peyzaj tasarımında en yaygın kullanım alanları, 49 türle çatı bahçelerinde belirlenirken, 31 türle yer koruma ve erozyon önleyiciler izlenmektedir. Çoğu sukkulent olan bu halofit türler hem sulak hem de kurak alanlara iyi adapte olduklarından gelecekte yaşanabilecek küresel 1sınma tehdidine karşı kurak ve çorak araziler için sürdürülebilir bir alternatif olacaktır. Bunun yanında çoğu endemik olan bu türlerin peyzaj tasarımında kullanılması aşırı tuzdan çoraklaşmış arazilerin restorasyonu, biyolojik çeşitliliğin korunması ve sürdürülebilir tarım uygulamaları için büyük önem taşımaktadır. 


\section{Introduction}

At the beginning of the 21 st century, as a result of global climate change, temperatures are rising, precipitation is decreasing and drought problems are emerging in the world. In many countries, the trends towards the use of natural plant species are increasing, and various regulations are being made in order to encourage the use of natural plant species by restricting the entry of foreign dwellers and organizing at different levels for this purpose. For instance, in the southern United States, landscape architects are using more sustainable design and management techniques, which include the expanded use of regional native plants (Robert et al., 2007). For this reason, the materials used in landscape designs are required to be replaced by natural plant species which are the most suitable materials for our country conditions. Prioritizing aesthetic approach instead of ecological approach in landscape design studies reveals designs that are not compatible with ecological and climatic conditions and, natural structure. Also, these are costly and unsustainable (Korkut et al., 2017). As a result of decreasing water resources and deteriorating the quality of the water resources, it is necessary to use the natural species belonging to the region, especially the arid and salt tolerating plants.

There are 954 million hectares of land in the world affected by salt and being less productive (Akbaş and Güvensen, 2001; Sözeri and Tipırdamaz, 2001). In Turkey, approximately 1.5 million hectares of suitable for irrigation area have salinity and alkalinity, 2.8 million hectares have drainage problems (Deliboran and Savran, 2015). Salt marshes are scattered on the surface of the earth and covered about $10 \%$ of the world's terrestrial areas. Salty soils are commonly found in arid landscapes where evapotranspiration exceeds precipitation throughout most of the year (Aschenbach, 2006; Çakır et al., 2010). A species capable of tolerating $0.5 \%$ or more $\mathrm{NaCl}$ are regarded as halophytes (Chapman, 1974) and have an excellent potential for landscaping (Martin, 1986). Halophytes, representing $\sim 1 \%$ of the world's flora, are plants that can grow and complete their entire life cycles in environments with high concentrations of electrolytes in the root medium (Flowers et al., 1977; Flowers and Colmer, 2008; Norman et al., 2013).

There are about 6.000 halophytic plant species throughout the world, 700 in the Mediterranean climate zone (Choukr-Allah, 1991), 406 in Turkey (Yaprak and Tuğ, 2006). There is potentially large number of halophytes in Central Anatolia, which is considered one of the Turkey's major centres of plant biodiversity. In Central Anatolia, Salt Lake closed basin and other salt marshes are a gene bank for halophytic species. It is inevitable that these genes, which are contained in the species resistant to salt and drought, will be used in agricultural applications in the future. Families such as Frankeniaceae, Tamaricaceae, Zygophyllaceae, Cyperaceae and Plumbaginaceae, especially the Chenopodiaceae family, contain species resistant to high salt concentrations (O'leary, 1994; Sözeri and Tıpırdamaz, 2001). Due to aesthetic appearance of halophytic plants in their natural recreation areas and importance as food sources, it has become more important to cultivate in their own habitats as landscaping and pasture plants rather than reclamation of salt-affected lands. The demand for landscaping plantings with low water requirement (Clark and Matheny, 1998) and low cost management is increasing, and this has led to halophytes and other natural species being used for revegetation or preserving soils with little plant cover (Cassaniti and Romano, 2011). Halophytes adapt to the salinity during the evolutionary processes and have changed their morphological and physiological structures accordingly. Therefore, halophytes can be a wide area of use in landscape design (Abd El-Ghani, 2000), because some plants that prefer salty conditions live in freshwater conditions. It is necessary to know the priority habitats and general characteristics of the plant species to be used in landscape design and to determine the characteristics of these species and their usage potential in planting studies (Deniz and Şirin, 2005). These plants naturally grown in saline soils are plants with different root depths. They are able to cover the soil surface, fast growing, resistant to drought and temperature and also able to stay green for a long time. For this reason, the use of these halophytes in salt-affected soils will be an appropriate alternative.

Nevertheless, there is not yet sufficient research on the use of native halophyte plants of Central Anatolia in landscape design. The goal of the presented study is to define some halophytes (Picture 1) and landscape values naturally grown in saline habitats in Central Anatolia. It is also encouraging the use of native plant species for sustainable landscape practices.

\section{Material and Methods}

Within the scope of the research, during the vegetation period, field studies were made in the saline habitats of Konya, Ankara, Aksaray and Nevşehir in the years of 2016 and 2017, and photographs of plant species were taken and herbarium specimens were collected. The identification of plant species and determination of Turkish names was made by Davis (1965-1985), Menemen and Hamzaoğlu (2000), Tugay et al. (2010), Güner et al. (2012), and Vural et al. (2012). The life forms of the species were classified according to Ellenberg and Mueller-Dombois (1967). The aesthetic properties of the plants were determined by taking into consideration the beauty of flowers, leaves, fruits and the beauty of the body's measure, colour, form and texture. On the other hand, visual and physical benefits, climate, noise and erosion were taken into consideration in functional use (Tanay et al., 2006). The aesthetic values and landscape use of species were made according to Eroğlu et al. (2013), Hansen and Alvarez (2010), Jackson (1914), Koç and Güneş (1998), and Thomas (2004).

\section{Characteristics of The Study Area}

The most important salt marshes in Central Anatolia are Tuz, Seyfe and Yay Lakes (Sultansazlığı). Tuz Lake (905 m) is located between Konya, Ankara and Aksaray provinces. Seyfe Lake (1100 m) and Yay Lake (1072 m) are located in Kırşehir and Kayseri province, respectively. Most of the salt marshes in Central Anatolia are formed near the salt lakes and have no flow to outside from basin (Figure 1). 


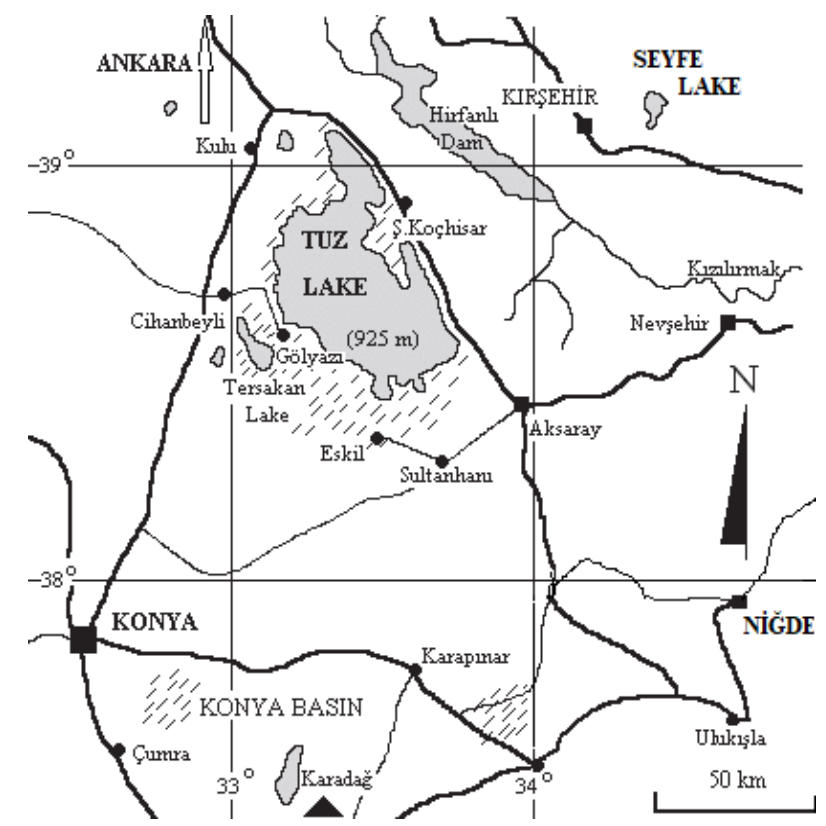

Figure 1 Some important salt marshes in Central Anatolia; Tuz Lake, Konya Basin and Seyfe Lake (modified from Hamzaoğlu and Aksoy, 2009)

The climatic data was obtained from meteorological stations of Cihanbeyli, Konya, Kırşehir and Aksaray located near the salt marshes of Central Anatolia. These salt marshes occupy the basins where the drainage is spoilt in the region. In the region, semi-arid, lower-very cold Mediterranean climate is dominant (Hamzaoğlu and Aksoy, 2009). Annual average temperature changes between 10 and $12^{\circ} \mathrm{C}$. The temperature is over $20^{\circ} \mathrm{C}$ during the summer season. The highest temperatures are seen in July and August in when the rain is minimum at the same time. In most part of the region, the annual precipitation is under $400 \mathrm{~mm}$. The arid season that begins in the end of June continues for 4-5 months (Hamzaoğlu and Aksoy, 2009). The salt lakes are nourished by few spring waters around and the rising base water in the winter and spring. Generally, saline, alkali and salt-alkali soils are found in the research area. During the rainy seasons (winter and spring), the rise of saltcontaining basal waters, floods and evaporation caused salt accumulation from the surface. $\mathrm{pH}$ generally varies between 7.5-8.5 due to the saturation of the soil with sodium and the presence of sodium carbonate in the soil solution (Hamzaoğlu and Aksoy, 2009; Furtana, 2010). Halophytes are rarely found in saline-alkaline soils, which have no agricultural value and considered barren soils (Anonymous, 1992). The annual average values of water saturation, $\mathrm{pH}$ mud, $\mathrm{EC}, \mathrm{Ca}^{++}, \mathrm{K}^{+}, \mathrm{HCO}_{3-}$ and $\mathrm{CEC}$ variables in Tuz Lake and its environs are the factors that change the soil salinity (Tuğ, 2006). These soils are alluvial hydromorphic and halomorphic soils composed of soluble $\mathrm{Na}^{+}, \mathrm{Ca}^{++}, \mathrm{K}^{+}$and $\mathrm{Mg}^{++}$cations and $\mathrm{Cl}^{-}, \mathrm{CO}_{3}{ }^{-}$, $\mathrm{NO}_{3}{ }^{-}, \mathrm{HCO}_{3}{ }^{-}$and $\mathrm{SO}_{4}^{--}$anions (Eyüpoğlu, 1999). Tuz Lake and Yay Lake, which have a rather rich flora and fauna have been severely degraded due to human pressure. Therefore, these two special lakes were declared as Special Environment Protection Area. On the other hand, Seyfe Lake was declared as a Nature Protection Area and taken under protection.

\section{Results and Discussion}

In the present study, 59 halophytic plant species belonging to 19 families and 38 genus that could be used in landscape design were identified from field studies and previous flora publications (Table 1a, 1b, 1c). The most representative family was the Asteraceae with 11 species, followed by Plumbaginaceae (9 species) and Chenopodiaceae (8 species). The genus Limonium from Plumbaginaceae family is well represented with 8 species. $15 \%$ of these plants (9 species) are therophyt (Annual), $85 \%$ are perennial (50 species); $51 \%$ were hemicryptophyte (30 species), $22 \%$ were chamaephyte (13 species), 6.7\% were geophyte (4 species) and 5\% hydrophyte species (Figure 2). The endemism rate is $42 \%$ (25 species). It was determined that 9 of the species could be used in natural and artificial water sides, 19 in roof and terrace gardens, 49 in rock gardens, 13 in flower partery, 31 for groundcover and erosion preventive, 13 in vertical gardens, 10 for exhibition and demonstration purposes, 9 as a border plant, and 7 for emphasis purposes (Table 1a, 1b, 1c).

Table (1a, 1b, 1c) may provide some understanding of the relationship between plant characteristics and their landscape uses as they appear in the habitats. 406 halophytic taxa are distributed in Turkey and 65 of them are endemic. Most of the inland saline habitats in Turkey are distributed in Central Anatolia, which is a closed basin, surrounded by mountains, especially Tuz Lake basin (Yaprak and Tuğ, 2006). According to Zahran (1982), $90 \%$ of the floristic composition of 30 community types and their members in Egyptian salt marshes are not only tolerant to saline soils but also to arid climates. All halophyte species might be of use in Central Anatolian landscapes where salinity and scarcity of water resources are major problems, because these plant species show resistance to salt and drought stress, high water use efficiency (Sanchez-Blanco et al 2002: Cassaniti and Romano 2011). The most representative family was the Asteraceae with 11 species, followed by Plumbaginaceae (9 species) and Chenopodiaceae (8 species) (Figure 2).

The genus Limonium from Plumbaginaceae family is well represented with 8 species (Table 1a, 1b, 1c), and most of them are preferred in landscape design due to lavender colored flowers. It is also remarkable that the majority of species in which ornamental characters, belong to the genus Limonium (Cassaniti and Romano, 2011), and some species including Limonium spp. are already grown commercially (Zia et al. 2008).

Halophytes have the potential to be used for many purposes, the most ideal areas where plants can be used; it has been attempted to be determined by taking into consideration the characteristics of plant and plant growth environment. In the present study, most commonly used species in landscape design are 49 of species in rock gardens, 31 for groundcover and erosion preventive, 19 in roof and terrace gardens, and 13 in flower partery (Figure 3). Halophytes are classified as (1) Euhalophytes which accumulate the salt in the old plant organs and are very resistant to the increase of the salt in the environment; those with succulent leaves (Salsola L., Suaeda Forssk. ex J.F.GmeI.) and succulent stems (Salicornia L., Halocnemum M.Bieb., Halostachys C.A.Mey.), (2) 
recretohalophytes which secrete salt from stem by salt glands (Cressa L., Frankenia L., Limonium Mill., Tamarix L.) or salt pouch (Atriplex L., Halimione Aellen, Mesembryanthemum L.), (3) pseudohalofites (such as Juncus L. and rosette leave plants) that can partly divide

the salt in their tissues and act as part of the true halophytes, and (4) nonhalophytes, which can develop in the appropriate seasons in saline fields or be selective against $\mathrm{Na}+$ and $\mathrm{Cl}^{-}$(Breckle, 1983).

Table 1a List of some halophytic plants and their landscape values in Central Anatolia.

\begin{tabular}{|c|c|c|c|c|c|c|c|c|c|c|}
\hline \multirow{2}{*}{ Scientific name of taxa $(\mathrm{TN})$} & \multirow{2}{*}{$\mathrm{LF} / \mathrm{FP}$} & \multicolumn{6}{|c|}{ Aesthetic values } & \multirow{2}{*}{ ULP } & \multirow{2}{*}{ A } & \multirow{2}{*}{ LRA } \\
\hline & & AS (V/H) & $\mathrm{PF}$ & $\mathrm{T}$ & LC & FC & E & & & \\
\hline \multicolumn{11}{|c|}{ Amaryllidaceae } \\
\hline $\begin{array}{l}\text { Allium scabriflorum Boiss (Tuz } \\
\text { körmeni) }\end{array}$ & G/6-7 & $10-30 / 5-10$ & $\mathrm{Ve}$ & $\mathrm{mt}$ & gr & $\begin{array}{c}\text { vi-bl, } \\
\text { pu }\end{array}$ & - & $\mathrm{B}, \mathrm{C}, \mathrm{F}$ & $700-1700$ & $\begin{array}{c}\text { Ankara, Konya, } \\
\text { Niğde; Tuz Lake }\end{array}$ \\
\hline $\begin{array}{l}\text { Allium sieheanum Kollmann } \\
\text { (Boncuk sarımsak) }\end{array}$ & $\mathrm{G} / 7-8$ & $15-25 / 5-10$ & $\mathrm{Ve}$ & st & gr & pi-pu & - & A & $900-1200$ & $\begin{array}{c}\text { Ankara; Tuz Lake, } \\
+\quad \text { Konya; Bolluk Lake, } \\
\text { Niğgde } \\
\end{array}$ \\
\hline \multicolumn{11}{|c|}{ Amaranthaceae } \\
\hline $\begin{array}{l}\text { Camphorosma monspeliaca L. } \\
\text { subsp. lessingii (Litv.) Aellen } \\
\text { (Çorak döşeği) }\end{array}$ & $\mathrm{Ch} / 6-8$ & $10-50 / 20-40$ & $\mathrm{Ve}$ & st & gr & - & - & $\mathrm{CD}$ & $1-1400$ & $\begin{array}{c}\text { Konya; Cihanbeyli- } \\
\text { Yavşan Tuzlası }\end{array}$ \\
\hline $\begin{array}{l}\text { Camphorosma monspeliaca L. } \\
\text { subsp. monspeliaca (Ezgen) }\end{array}$ & $\mathrm{Ch} / 6-8$ & $10-50 / 20-40$ & $\mathrm{Ve}$ & st & gr & - & - & C,D & $1-3000$ & $\begin{array}{c}\text { Konya; kaşınhanı, } \\
\text { - } \begin{array}{c}\text { Cihanbeyli-Tersakan } \\
\text { Lake, Aksaray }\end{array} \\
\end{array}$ \\
\hline \multicolumn{11}{|c|}{ Asteraceae } \\
\hline $\begin{array}{l}\text { Achillea aleppica DC. subsp. } \\
\text { zederbaueri (Akbaşl1) }\end{array}$ & $\mathrm{H} / 5-7$ & $5-15 / 5-15$ & $\mathrm{Ve}$ & $\mathrm{dt}$ & gr & wh & - & $\begin{array}{l}\mathrm{C}, \mathrm{E}, \\
\mathrm{G}, \mathrm{H}\end{array}$ & $660-1380$ & $\begin{array}{c}\text { Konya; Yavşantuzlası, } \\
\text { Nevşehir, Niğde, } \\
\text { Karaman }\end{array}$ \\
\hline $\begin{array}{l}\text { Achillea goniocephala Boiss. \& } \\
\text { Balansa (Hırpkesti) }\end{array}$ & $\mathrm{H} / 6-8$ & $15-40 / 10-15$ & $\mathrm{Ve}$ & $\mathrm{mt}$ & pgr & wh & - & $\mathrm{C}, \mathrm{E}, \mathrm{H}$ & $1300-1900$ & $+\quad \begin{array}{c}\text { Niğde; Bereketli } \\
(\text { Çamardı) }\end{array}$ \\
\hline $\begin{array}{l}\text { Anthemis fumariifolia Boiss. } \\
\text { (Çorak papatyas1) }\end{array}$ & Th/5-6 & $10-25 / 15-25$ & $\mathrm{Ve}$ & st & gr & wh & - & $\mathrm{A}, \mathrm{C}$ & $870-1675$ & $\begin{array}{l}\text { Ankara, Aksaray, } \\
\quad \text { Konya; Tuz Lake }\end{array}$ \\
\hline $\begin{array}{l}\text { Artemisia santonicum } \mathrm{L} \text {. subsp. } \\
\text { santonicum. (Deniz yavşanı) }\end{array}$ & $\mathrm{Ch} / 7-10$ & $-60 / 30$ & $\mathrm{Ve}$ & st & pgr & ye & - & $\mathrm{C}, \mathrm{G}$ & $0-1300$ & $\begin{array}{c}\text { Ankara; } \\
\text { - } \quad \text { Şerefikoçhiar-Tuz } \\
\text { Lake, Konya; Ereğli }\end{array}$ \\
\hline $\begin{array}{l}\text { Helichrysum noeanum Boiss. } \\
\text { (Gülazar) }\end{array}$ & $\mathrm{H} / 6-8$ & $8-17 / 5-15$ & Co & $\mathrm{dt}$ & pgr & ye & - & C,D & $900-1500$ & $\begin{array}{c}\text { Konya, Ankara; } \\
+\quad \begin{array}{c}\text { Şereflikoçhisar, Tuz } \\
\text { Lake }\end{array}\end{array}$ \\
\hline $\begin{array}{l}\text { Inula aucherana DC. (Çorak } \\
\text { andizotu) }\end{array}$ & $\mathrm{H} / 7-9$ & $15-75 / 5-15$ & $\mathrm{Ve}$ & st & gr & ye & - & $\mathrm{C}, \mathrm{G}$ & $550-1900$ & $\begin{array}{c}\text { Konya, Niğde, } \\
\text { Aksaray }\end{array}$ \\
\hline $\begin{array}{l}\text { Scorzonera hieraciifolia } \text { Hayek } \\
\text { (C1b1l tekesakalı) }\end{array}$ & $\mathrm{H} / 5-6$ & $5-15 / 5-10$ & $\mathrm{Cr}$ & $\mathrm{mt}$ & $\mathrm{gr}$ & ye & - & $\begin{array}{c}\mathrm{C}, \mathrm{D}, \\
\mathrm{H}, \mathrm{I}\end{array}$ & $800-1350$ & $+\begin{array}{c}\text { Konya; Cihanbeyli, } \\
\text { Aksaray }\end{array}$ \\
\hline $\begin{array}{l}\text { Scorzonera parviflora Jacq. } \\
\text { (Çatalkök) }\end{array}$ & $\mathrm{H} / 5-9$ & $5-35 / 5-15$ & $\mathrm{Ve}$ & $\mathrm{dt}$ & gr & ye & - & $\mathrm{A}, \mathrm{C}, \mathrm{H}$ & $800-1500$ & $\begin{array}{c}\text { Konya; Tuz Lake, } \\
\text { - Kırşehir; Seyfe Lake, } \\
\text { Niğde }\end{array}$ \\
\hline $\begin{array}{l}\text { Taraxacum farinosum Rausskn. } \\
\text { \&Bornm. (Çıtllk) }\end{array}$ & $\mathrm{H} / 6-9$ & $20-30 / 5-10$ & $\begin{array}{c}\mathrm{Co}, \\
\mathrm{Cl}\end{array}$ & $\mathrm{dt}$ & pgr & ye & - & C,D & $800-1200$ & $\begin{array}{c}\text { Konya; Kırkışla } \\
+\quad \text { Acıtuz Lake, Niğde; } \\
\text { Tuz Lake }\end{array}$ \\
\hline $\begin{array}{l}\text { Taraxacum mirabile Wagenitz } \\
\text { (Akcirtlık) }\end{array}$ & $\mathrm{H} / 3-5$ & $10-20 / 5-15$ & $\mathrm{Cr}$ & $\mathrm{dt}$ & gr & wh & - & $\mathrm{C}, \mathrm{D}, \mathrm{H}$ & $800-1300$ & $+\quad$ Niğde;Tuz Lake \\
\hline $\begin{array}{l}\text { Tripleurospermum parviflorum } \\
\text { (Willd.) Pobed. (Beybunik) }\end{array}$ & Th/3-6 & $4-35 / 10-25$ & $\mathrm{Ve}$ & st & gr & wh & - & $\mathrm{C}, \mathrm{H}$ & $30-1180$ & $\begin{array}{c}\text { Konya; Cihanbeyli- } \\
\text { - } \quad \text { Tuz Lake, Nevşehir; } \\
\text { Göreme }\end{array}$ \\
\hline \multicolumn{11}{|c|}{ Boraginaceae } \\
\hline $\begin{array}{l}\text { Onosma halophila Boiss. \& } \\
\text { Heldr (Ac1 emcek) }\end{array}$ & $\mathrm{H} / 6-7$ & $15-25 / 5-15$ & $\mathrm{Ve}$ & $\mathrm{mt}$ & pgr & $\mathrm{cr}$ & - & $\begin{array}{l}\mathrm{C}, \mathrm{E}, \\
\mathrm{G}, \mathrm{H}\end{array}$ & $900-1050$ & $+\begin{array}{c}\text { Konya; Cihanbeyli, } \\
\text { Çumra }\end{array}$ \\
\hline \multicolumn{11}{|c|}{ Brassicaceae } \\
\hline $\begin{array}{l}\text { Iberis halophila Vural } \\
\& \text { H.Duman (Tuz beğendiotu) }\end{array}$ & $\mathrm{H} / 6-7$ & $4-13 / 5-10$ & Co & $\mathrm{dt}$ & gr & wh & - & $\begin{array}{c}\mathrm{C}, \mathrm{D}, \\
\mathrm{H}, \mathrm{I}\end{array}$ & $930-980$ & $\begin{array}{c}\text { Konya; Cihanbeyli- } \\
\text { Tersakan Lake, } \\
\text { Aksaray-Eskil }\end{array}$ \\
\hline $\begin{array}{l}\text { Lepidium cartilagineum } \\
\text { (J.Mayer) TheIl. subsp. } \\
\text { caespitosum TheIl. (Yatık tere) }\end{array}$ & $\mathrm{H} / 4-6$ & $7-12 / 10-20$ & $\mathrm{Ve}$ & $\mathrm{dt}$ & gr & wh & - & $\mathrm{C}$ & $900-1300$ & $\begin{array}{c}\text { Konya-Kadınhanı, } \\
\text { Ankara-Tuz Lake, } \\
\text { Nevşehir }\end{array}$ \\
\hline \multicolumn{11}{|c|}{ Caryophyllaceae } \\
\hline $\begin{array}{l}\text { Dianthus aydogdui Menemen \& } \\
\text { Hamzaoğlu (Ay karanfili) }\end{array}$ & $\mathrm{Th} / 6-7$ & $3-15 / 10-15$ & $\mathrm{Ve}$ & $\mathrm{dt}$ & $\mathrm{gr}$ & pi & - & $\begin{array}{l}\mathrm{C}, \mathrm{E}, \\
\mathrm{G}, \mathrm{H}\end{array}$ & 950 & $\begin{array}{c}\text { Aksaray; Ulukışla, } \\
\text { Tuz Lake }\end{array}$ \\
\hline $\begin{array}{l}\text { Silene salsuginea Hub.-Mor. } \\
\text { (Çorak nak1l1) }\end{array}$ & $\mathrm{Ch} / 7-8$ & $25 / 10-30$ & $\mathrm{Ve}$ & $\mathrm{mt}$ & pgr & wh & - & $\mathrm{C}$ & $900-900$ & $+\quad$ Konya; Tuz Lake \\
\hline $\begin{array}{l}\text { Spergularia media (L.) C.Presl } \\
\text { (Kuş remilotu) }\end{array}$ & $\mathrm{H} / 5-8$ & $5-40 / 10-30$ & $\mathrm{Cr}$ & $\mathrm{mt}$ & gr & pvi & - & C,D & $0-950$ & - Konya-Yavşan Tuzlası \\
\hline
\end{tabular}


Table 1b List of some halophytic plants and their landscape values in Central Anatolia.

\begin{tabular}{|c|c|c|c|c|c|c|c|c|c|c|}
\hline \multirow{2}{*}{ Scientific name of taxa (TN) } & \multirow{2}{*}{$\mathrm{LF} / \mathrm{FP}$} & \multicolumn{6}{|c|}{ Aesthetic values } & \multirow{2}{*}{ ULP } & \multirow{2}{*}{ A } & \multirow{2}{*}{ LRA } \\
\hline & & $\mathrm{AS}(\mathrm{V} / \mathrm{H})$ & PF & $\mathrm{T}$ & LC & $\mathrm{FC}$ & $\mathrm{E}$ & & & \\
\hline \multicolumn{11}{|c|}{ Chenopodiaceae } \\
\hline $\begin{array}{l}\text { Chenopodium chenopodioides } \\
\text { (L.) Aellen (Kaz sirkeni) }\end{array}$ & Th/5-8 & $15-50 / 80$ & $\mathrm{Cr}$ & $\mathrm{dt}$ & & ye-br & - & $\mathrm{C}, \mathrm{D}, \mathrm{F}$ & $1-900$ & Konya; Kayacık \\
\hline $\begin{array}{l}\text { Halimione portulacoides (L.) } \\
\text { Aellen (Koca betne) }\end{array}$ & $\mathrm{Ch} / 6-8$ & $75 / 30-80$ & $\mathrm{Cr}$ & $\mathrm{dt}$ & gr & na & Ev & $\begin{array}{c}\text { A,C,D } \\
\text { H,I }\end{array}$ & $0-900$ & $\begin{array}{c}\text { Konya; Cihanbeyli- } \\
\text { Yavşan Tuzlası }\end{array}$ \\
\hline $\begin{array}{l}\text { Halimione verrucifera Aellen } \\
\text { (Betne) }\end{array}$ & $\mathrm{Ch} / 6-8$ & -50 & Co & $\mathrm{dt}$ & gr & na & Ev & C,D & $1-1200$ & $\begin{array}{c}\text { Konya, Aksaray; Tuz } \\
\text { Lake }\end{array}$ \\
\hline $\begin{array}{l}\text { Halocnemum strobilaceum } \\
\text { M.Bieb. (Çuvan) }\end{array}$ & Chs/7-9 & $10-35 / 20-40$ & $\begin{array}{l}\mathrm{Ve}, \\
\mathrm{Cl}\end{array}$ & $\mathrm{dt}$ & $\mathrm{gr}$ & na & Ev & $\begin{array}{c}\mathrm{C}, \mathrm{D}, \\
\mathrm{H}, \mathrm{I}\end{array}$ & $0-1200$ & $\begin{array}{c}\text { Konya; Cihanbeyli } \\
\text { - Bolluk Lake, Aksaray; } \\
\text { Tuz Lake }\end{array}$ \\
\hline $\begin{array}{l}\text { Microcnemum coralloides } \\
\text { (Loscos \& J.Pardo) Font Quer } \\
\text { (Şamdanotu) }\end{array}$ & Ths/5-7 & $5-10 / 10$ & $\mathrm{Ve}$ & $\mathrm{dt}$ & gr-re & na & Ev & $\begin{array}{l}\mathrm{B}, \mathrm{C}, \mathrm{D}, \\
\mathrm{H}, \mathrm{I}\end{array}$ & $940-100$ & $\begin{array}{c}\text { Konya; S. E. of Tuz } \\
\text { Lake }\end{array}$ \\
\hline $\begin{array}{l}\text { Salicornia perennans Wild. } \\
\text { (Yaşl1 geren) }\end{array}$ & Ths/5-7 & $\mathrm{Na} / 10-20$ & $\mathrm{Ve}$ & $\mathrm{dt}$ & $\begin{array}{l}\text { gr- } \\
\text { rpu }\end{array}$ & na & Ev & $\begin{array}{l}\mathrm{C}, \mathrm{G}, \\
\mathrm{H}, \mathrm{I}\end{array}$ & $900-1100$ & $\begin{array}{c}\text { Konya; Kırkışla- } \\
\text { - Acıtuz Lake, Aksaray- } \\
\text { Ş. Koçhisar }\end{array}$ \\
\hline $\begin{array}{l}\text { Salsola crassa M.Bieb. (Etli } \\
\text { soda) }\end{array}$ & Ths/5-7 & $20-45 / 25-50$ & $\mathrm{Ve}$ & $\mathrm{mt}$ & $\mathrm{gr}$ & cr & - & $\mathrm{B}, \mathrm{C}$ & 1000 & $\begin{array}{c}\text { Konya; Cihanbeyli- } \\
\text { Yavşantuzlası, Niğde } \\
\end{array}$ \\
\hline $\begin{array}{l}\text { Salsola inermis Forssk. (Masum } \\
\text { sodaotu) }\end{array}$ & $\mathrm{Th} / 5-7$ & $20-50 / 35$ & $\mathrm{Cl}$ & $\mathrm{dt}$ & pgr & $\mathrm{cr}$ & - & $\mathrm{B}, \mathrm{C}$ & $900-950$ & $\begin{array}{c}\text { Ankara; Salt Lake, } \\
\text { Aksaray; Sultanhanı } \\
\end{array}$ \\
\hline \multicolumn{11}{|c|}{ Cyperaceae } \\
\hline $\begin{array}{l}\text { Bolboschoenus maritimus (L.) } \\
\text { Palla subsp. maritimus } \\
\text { (Sandalye sazı) }\end{array}$ & Cry/5-9 & $60-100 / 10-30$ & $\mathrm{Ve}$ & st & gr & re-br & - & A & $1-2000$ & $\begin{array}{c}\text { Konya; Cihanbeyli, } \\
\text { - Bolluk Lake, kırşehir; } \\
\text { Seyfe Lake } \\
\end{array}$ \\
\hline \multicolumn{11}{|c|}{ Fabaceae } \\
\hline $\begin{array}{l}\text { Astragalus karamasicus Boiss. } \\
\text { \& Bal. (Korumaz geveni) }\end{array}$ & $\mathrm{H} / 6-7$ & $10-20 / 15-35$ & $\mathrm{Cl}$ & $\mathrm{dt}$ & & pi,pu & - & C,D & $450-2060$ & $+\begin{array}{c}\text { Niğde, Konya-Tuz } \\
\text { Lake }\end{array}$ \\
\hline $\begin{array}{l}\text { Astragalus ovalis Boiss. } \\
\& \text { Balansa (Tuz geveni) }\end{array}$ & $\mathrm{H} / 6$ & $-30 / 15-40$ & $\mathrm{Ve}$ & $\mathrm{mt}$ & gr & pi & - & $\mathrm{E}$ & $800-1400$ & $+\quad$ Konya; Tuz Lake \\
\hline $\begin{array}{l}\text { Lotus strictus Fisch. \& } \\
\text { C.A.Mey. (Böbrek otu) }\end{array}$ & $\mathrm{H} / 6-8$ & $10-50 / 30-80$ & $\mathrm{Cr}$ & $\mathrm{mt}$ & gr & $\mathrm{cr}$ & - & $\mathrm{D}$ & $500-1300$ & - Konya; Bolluk Lake \\
\hline \multicolumn{11}{|c|}{ Frankeniaceae } \\
\hline $\begin{array}{l}\text { Frankenia hirsuta } \mathrm{L} \text {. } \\
\text { (Tülpembe) }\end{array}$ & $\mathrm{Ch} / \mathrm{NA}$ & $5-15 / 10-20$ & $\mathrm{Cr}$ & & pgr & pi & - & $\begin{array}{c}\mathrm{C}, \mathrm{D}, \\
\mathrm{H}, \mathrm{I}\end{array}$ & $0-1400$ & - W., E. and C. Anatolia \\
\hline $\begin{array}{l}\text { Frankenia pulverulenta } \\
\text { L.(Çorak pembe) }\end{array}$ & $\mathrm{Th} / 7-8$ & $30 / 30$ & $\mathrm{Cr}$ & $\mathrm{dt}$ & gr & pi & - & $\mathrm{C}, \mathrm{D}, \mathrm{H}$ & $1-1000$ & $\begin{array}{c}\text { Konya; Cihanbeyli- } \\
\text { Tersakan Lake }\end{array}$ \\
\hline $\begin{array}{l}\text { Frankenia salsuginea Adıgüzel } \\
\& \text { Aytaç (Hoş pembe) }\end{array}$ & $\mathrm{G} / 6-7$ & $25 / 35$ & $\mathrm{Cr}$ & $\mathrm{dt}$ & $\mathrm{gr}$ & pi,pu & - & $\mathrm{B}, \mathrm{C}, \mathrm{D}$ & 1000 & $+\begin{array}{c}\text { Konya; Cihanbeyli } \\
\text { Tersakan Lake }\end{array}$ \\
\hline \multicolumn{11}{|c|}{ Iridaceae } \\
\hline $\begin{array}{l}\text { Gladiolus halophilus Boiss. \& } \\
\text { Heldr. (Çorak k1liçotu) }\end{array}$ & G/6-7 & $25-55 / 20$ & $\mathrm{Ve}$ & st & gr & pi & - & $\begin{array}{l}\mathrm{A}, \mathrm{B}, \\
\mathrm{E}, \mathrm{F}\end{array}$ & $900-1200$ & Konya; Tuz Lake \\
\hline \multicolumn{11}{|c|}{ Juncaceae } \\
\hline $\begin{array}{l}\text { Juncus heldreichianus T. Marsson } \\
\text { ex ParI. subsp. orientalis } \\
\text { Snogerup (Kisa dombay) }\end{array}$ & Cry/5-7 & $30-70 / 15-40$ & $\mathrm{Ve}$ & st & $\mathrm{gr}$ & na & Ev & A & $800-1830$ & $\begin{array}{l}\text { Konya; Karapınar, } \\
\text { Obruk }\end{array}$ \\
\hline $\begin{array}{l}\text { Juncus maritimus Lam. } \\
\text { (Peygamber k1lıc1) }\end{array}$ & Cry/5-7 & $\mathrm{Ot} / 15-40$ & $\mathrm{Ve}$ & $\mathrm{mt}$ & gr & na & Ev & A & $0-1050$ & $\begin{array}{l}\text { Konya; Karapınar, } \\
\text { Meke Salt Lake }\end{array}$ \\
\hline \multicolumn{11}{|c|}{ Lamiaceae } \\
\hline $\begin{array}{l}\text { Salvia halophila Hedge (Tuz } \\
\text { şalbası) }\end{array}$ & $\mathrm{H} / 8-10$ & $-50 / 10-20$ & $\mathrm{Ve}$ & $\mathrm{mt}$ & gr & li & - & $\begin{array}{l}\mathrm{C}, \mathrm{D}, \mathrm{E}, \\
\mathrm{G}, \mathrm{H}, \mathrm{I}\end{array}$ & $950-1000$ & $\begin{array}{c}\text { Niğde, Aksaray, } \\
+\quad \text { Konya- S. of Tuz } \\
\text { Lake, Bolluk Like } \\
\end{array}$ \\
\hline $\begin{array}{l}\text { Thymus leucostomus Hausskn. } \\
\& \text { velen. (Ana kekik) }\end{array}$ & $\mathrm{Ch} / 5-7$ & $5-20 / 10-40$ & $\mathrm{Cr}$ & $\mathrm{mt}$ & gr & wh & - & $\mathrm{D}, \mathrm{E}, \mathrm{I}$ & $670-1600$ & $+\begin{array}{c}\text { Ankara, } 15 \mathrm{~km} \text { South } \\
\text { of Konya }\end{array}$ \\
\hline \multicolumn{11}{|c|}{ Linaceae } \\
\hline $\begin{array}{l}\text { Linum ertugrulii O.Tugay, } \\
\text { Y.Bağcı \& T.Uysal (Bey keteni) }\end{array}$ & $\mathrm{H} / 6-8$ & $5-15 / 10-25$ & $\mathrm{Cl}$ & $\mathrm{dt}$ & gr & ye & - & $\mathrm{C}, \mathrm{D}, \mathrm{H}$ & 920 & $\begin{array}{c}\text { Konya- Cihanbeyli- } \\
+\quad \text { Tuz Lake, Gölyazı, } \\
\text { Eskil }\end{array}$ \\
\hline $\begin{array}{l}\text { Linum seljukorum P.H.Davis } \\
\text { (Bolluk keteni) }\end{array}$ & $\mathrm{H} / 8-9$ & $6-20 / 10-30$ & $\mathrm{Cl}$ & $\mathrm{mt}$ & gr & bl & - & $\mathrm{C}, \mathrm{E}, \mathrm{H}$ & 1000 & $\begin{array}{c}\text { Konya; Kaşınhanı, } \\
\text { Cihanbeyli-Bolluk } \\
\text { Lake }\end{array}$ \\
\hline \multicolumn{11}{|c|}{ Plantaginaceae } \\
\hline $\begin{array}{l}\text { Plantago crassifolia Forssk. } \\
\text { (Nasırliyaprak) }\end{array}$ & $\mathrm{H} / 5-10$ & $10-30 / 10-30$ & $\begin{array}{l}\mathrm{Ve}, \\
\mathrm{Cl}\end{array}$ & & gr & na & Ev & $\begin{array}{c}\mathrm{C}, \mathrm{D}, \\
\mathrm{H}, \mathrm{I}\end{array}$ & $1-900$ & $\begin{array}{c}\text { Aksaray; S.E. of Tuz } \\
\text { Lake }\end{array}$ \\
\hline Plantago maritina L. (Y1landili) & $\mathrm{H} / 5-8$ & $15-40 / 15-30$ & $\begin{array}{l}\text { Ve, } \\
\mathrm{Cl}\end{array}$ & $\mathrm{dt}$ & gr & na & Ev & $\begin{array}{c}\mathrm{B}, \mathrm{C}, \mathrm{D}, \\
\mathrm{H}, \mathrm{I}\end{array}$ & $1-2400$ & $\begin{array}{c}\text { Konya; Cihanbeyli- } \\
\text { Acıgöl }\end{array}$ \\
\hline
\end{tabular}


Table 1c List of some halophytic plants and their landscape values in Central Anatolia.

\begin{tabular}{|c|c|c|c|c|c|c|c|c|c|c|}
\hline \multirow{2}{*}{ Scientific name of taxa (TN) } & \multirow{2}{*}{$\mathrm{LF} / \mathrm{FP}$} & \multicolumn{6}{|c|}{ Aesthetic values } & \multirow{2}{*}{ ULP } & \multirow{2}{*}{ A } & \multirow{2}{*}{ LRA } \\
\hline & & AS $(\mathrm{V} / \mathrm{H})$ & $\mathrm{PF}$ & $\mathrm{T}$ & LC & $\mathrm{FC}$ & $\mathrm{E}$ & & & \\
\hline \multicolumn{11}{|c|}{ Plumbaginaceae } \\
\hline $\begin{array}{l}\text { Acantholimon halophilum } \\
\text { Bokhari (Kirpiotu) }\end{array}$ & $\mathrm{Ch} / 6-6$ & $10-30 / 15-35$ & $\mathrm{Cl}$ & $\mathrm{dt}$ & & pi & & $\begin{array}{l}\text { B,C,D, } \\
\text { H,I }\end{array}$ & $900-1100$ & $+\begin{array}{c}\text { Konya; Cihanbeyli- } \\
\text { Acituz Lake }\end{array}$ \\
\hline $\begin{array}{l}\text { Limonium anatolicum Hedge } \\
\text { (Yer kuduzotu) }\end{array}$ & $\mathrm{H} / 6-9$ & $\mathrm{Na} / \mathrm{na}$ & $\mathrm{Cl}$ & $\mathrm{dt}$ & gr & li & - & $\mathrm{C}, \mathrm{D}$ & $900-1000$ & $\begin{array}{c}\text { Konya; Kaşınhanı, } \\
+\quad \text { Tuz Lake, Aksaray; } \\
\text { Sultansazlığı }\end{array}$ \\
\hline $\begin{array}{l}\text { Limonium gmelinii (Willd.) } \\
\text { Kuntze (Çardak süpürgesi) }\end{array}$ & $\mathrm{H} / 5-10$ & $30-60 / 30-40$ & $\mathrm{Ve}$ & $\mathrm{mt}$ & gr & bl-vi & - & $\begin{array}{l}\mathrm{A}, \mathrm{C}, \mathrm{E} \\
\mathrm{F}, \mathrm{G}\end{array}$ & $1-1450$ & $\begin{array}{c}\text { Konya- Kaşınhanı, } \\
\text { Niğde, Ankara. }\end{array}$ \\
\hline $\begin{array}{l}\text { Limonium bellidifolium (Gouan) } \\
\text { Dumort. (Hoş kuduzotu) }\end{array}$ & $\mathrm{H} / 6-9$ & $20 / 20-40$ & $\mathrm{Cl}$ & st & gr & li-vi & - & $\mathrm{C}, \mathrm{D}, \mathrm{E}$ & $0-1010$ & $\begin{array}{c}\text { Konya; } 26 \mathrm{~km} \mathrm{S.} \mathrm{of} \\
\text { Cihanbeyli }\end{array}$ \\
\hline $\begin{array}{l}\text { Limonium iconicum (Boiss. \& } \\
\text { Heldr.) Kuntze (Konya } \\
\text { kuduzotu) }\end{array}$ & $\mathrm{H} / 6-9$ & $\mathrm{Na} / \mathrm{na}$ & $\mathrm{Cl}$ & $\mathrm{mt}$ & gr & bl-vi & - & $\mathrm{C}, \mathrm{D}, \mathrm{E}$ & $900-1040$ & $\begin{array}{c}\text { Konya, Niğde, } \\
+\quad \text { Aksaray, Kayseri, } \\
\text { Ankara }\end{array}$ \\
\hline $\begin{array}{lrr}\text { Limonium } & \text { lilacinum } & \text { (Boiss. \& } \\
\text { Balansa) } & \text { Wagenitz } & \text { (Çorak } \\
\text { lavantas1) } & & \end{array}$ & $\mathrm{H} / 6-9$ & $20-30 / 20-30$ & $\mathrm{Cl}$ & st & gr & vi & - & $\mathrm{C}, \mathrm{D}, \mathrm{F}$ & $900-1200$ & $\begin{array}{l}\text { Konya; Kaşınhanı, } \\
\text { Aksaray; Tuz Lake }\end{array}$ \\
\hline $\begin{array}{l}\text { Limonium globuliferum (Boiss. } \\
\& \text { Heldr.) Kuntze (Boncuk } \\
\text { devekulağ } 1 \text { ) }\end{array}$ & $\mathrm{H} / 6-9$ & $15-30 / 15-25$ & $\mathrm{Cl}$ & $\mathrm{dt}$ & gr & pvi & - & $\mathrm{C}, \mathrm{F}$ & $900-1100$ & $\begin{array}{l}\text { Aksaray, Konya- } \\
\text { Kaşınhanı }\end{array}$ \\
\hline $\begin{array}{l}\text { Limonium pycnanthum (C. } \\
\text { Koch) O. Kuntze (Has } \\
\text { devekulağ } 1 \text { ) }\end{array}$ & $\mathrm{H} / 7-9$ & $20-30 / 20-30$ & $\mathrm{Ve}$ & st & gr & vi & - & $\mathrm{B}, \mathrm{C}$ & 1525 & W. of Kayseri \\
\hline $\begin{array}{l}\text { Limonium tamaricoides Bokhari } \\
\text { (Çorak kuduzotu) }\end{array}$ & $\mathrm{Ch} / 7-9$ & $15-25 / 25$ & $\mathrm{Ve}$ & st & gr & pvi & - & $\mathrm{C}$ & 950 & Aksaray \\
\hline \multicolumn{11}{|c|}{ Poaceae } \\
\hline $\begin{array}{l}\text { Crypsis aculeata (L.) Aiton } \\
\text { (Bakakotu) }\end{array}$ & Th/6-10 & $1-30 / 10-40$ & $\mathrm{Cl}$ & $\mathrm{dt}$ & & - & - & C,D & $1-1510$ & $\begin{array}{c}\text { Karaman-Ereğli, } \\
\text { Karapınar }\end{array}$ \\
\hline $\begin{array}{l}\text { Leymus cappadocicus (Boiss. \& } \\
\text { Bal.) Melderis (Tuz çavdarı) }\end{array}$ & $\mathrm{H} / 5-8$ & $25-50 / 10-20$ & $\mathrm{Ve}$ & $\mathrm{mt}$ & gr & - & - & $\mathrm{A}, \mathrm{F}$ & $900-1100$ & $\begin{array}{c}\text { Konya Niğde, Ankara; } \\
\text { Near Tuz Like }\end{array}$ \\
\hline $\begin{array}{l}\text { Puccinellia distans (Jacq.) ParI. } \\
\text { subsp. distans (Ayrık tuzçimi) }\end{array}$ & $\mathrm{H} / 7-8$ & $30-75 / 15-45$ & $\mathrm{Ve}$ & st & gr & - & - & G & $670-1350$ & $\begin{array}{c}\text { Konya; Cihanbeyli- } \\
\text { Yavşantuzlası }\end{array}$ \\
\hline \multicolumn{11}{|c|}{ Primulaceae } \\
\hline Glaux maritima L. (Sütlüot) & $\mathrm{H} / 5-8$ & $4-20 / 20-30$ & $\mathrm{Ve}$ & $\mathrm{dt}$ & gr & pi-li & - & B,C,D & $1-1720$ & $\begin{array}{l}\text { Kırşehir, Konya; } \\
\text { Bolluk Lake }\end{array}$ \\
\hline \multicolumn{11}{|c|}{ Tamaricaceae } \\
\hline $\begin{array}{l}\text { Reaumuria alternifolia (Labill.) } \\
\text { Britten (Kördiken) }\end{array}$ & $\mathrm{Ch} / 7-9$ & $30-50 / 40-90$ & $\mathrm{Cl}$ & st & gr & pi & - & $\begin{array}{l}\mathrm{C}, \mathrm{D}, \mathrm{E} \\
\mathrm{G}, \mathrm{H}, \mathrm{I}\end{array}$ & $900-1050$ & $\begin{array}{c}\text { Konya; Cihanbeyli- } \\
\text { - Tuz Lake, Aksaray; } \\
\text { Ulukışla }\end{array}$ \\
\hline $\begin{array}{l}\text { bbreviations: TN: Turklish name, L } \\
\text { hamaephyte, Chs: Succulent chamae } \\
\text { : Plant form (Ve: Vertical, Co: Com } \\
\text { lor and FC: Flower color (bl: blue, } \\
\text { Irple, pvi: pale violet, re: red, rpu: re } \\
\text { tificial water sides, B: For exhibition } \\
\text { or emphasis purposes, G: as a border } \\
\text { cality in the research area. }\end{array}$ & br: brow & $\begin{array}{l}\text { eeping, } \mathrm{Cl}: \mathrm{Cl} \\
\text { r: cream, gr: }\end{array}$ & & & & suc & & ure, $\mathrm{m}$ & ot available & $\begin{array}{l}\text { e, H: Hemicryptophyte, Ch: } \\
\text {, V: Vertical, H: Horizontal, } \\
\text { st: sparse texture), LC: Leaf } \\
\text { ggr: pale green, pi: pink, pu: } \\
\text { esign (A: On the natural and } \\
\text { entive, E: Flower Partery, F: } \\
\text { Endemic for Turkev LR }\end{array}$ \\
\hline
\end{tabular}
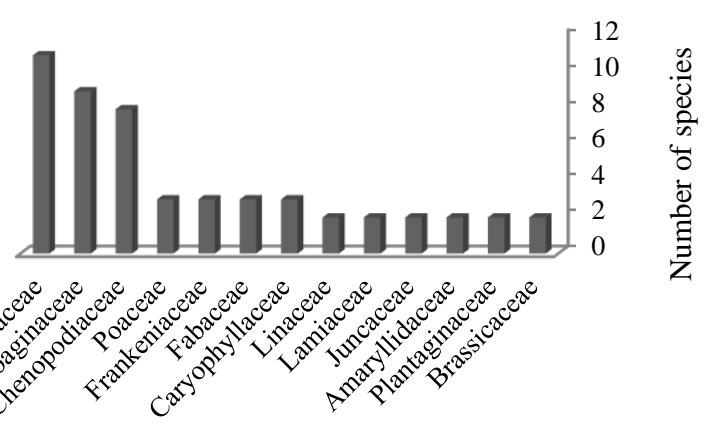

Figure 2 Families with the most halophyte species in landscape use

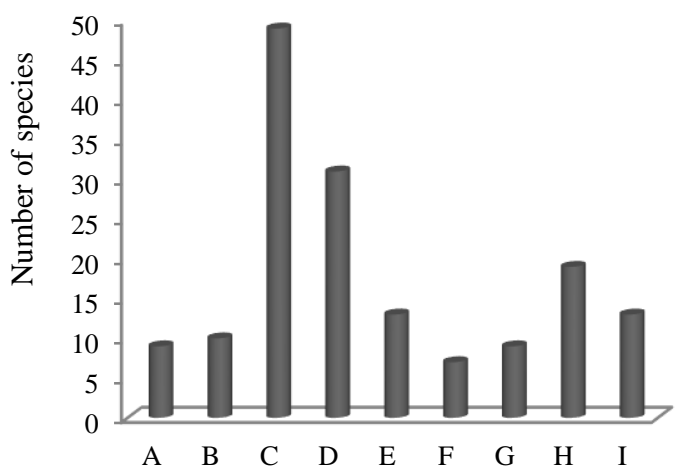

Figure 3 The landscape use of halophytes 
Picture 1 Photos of some halophytes
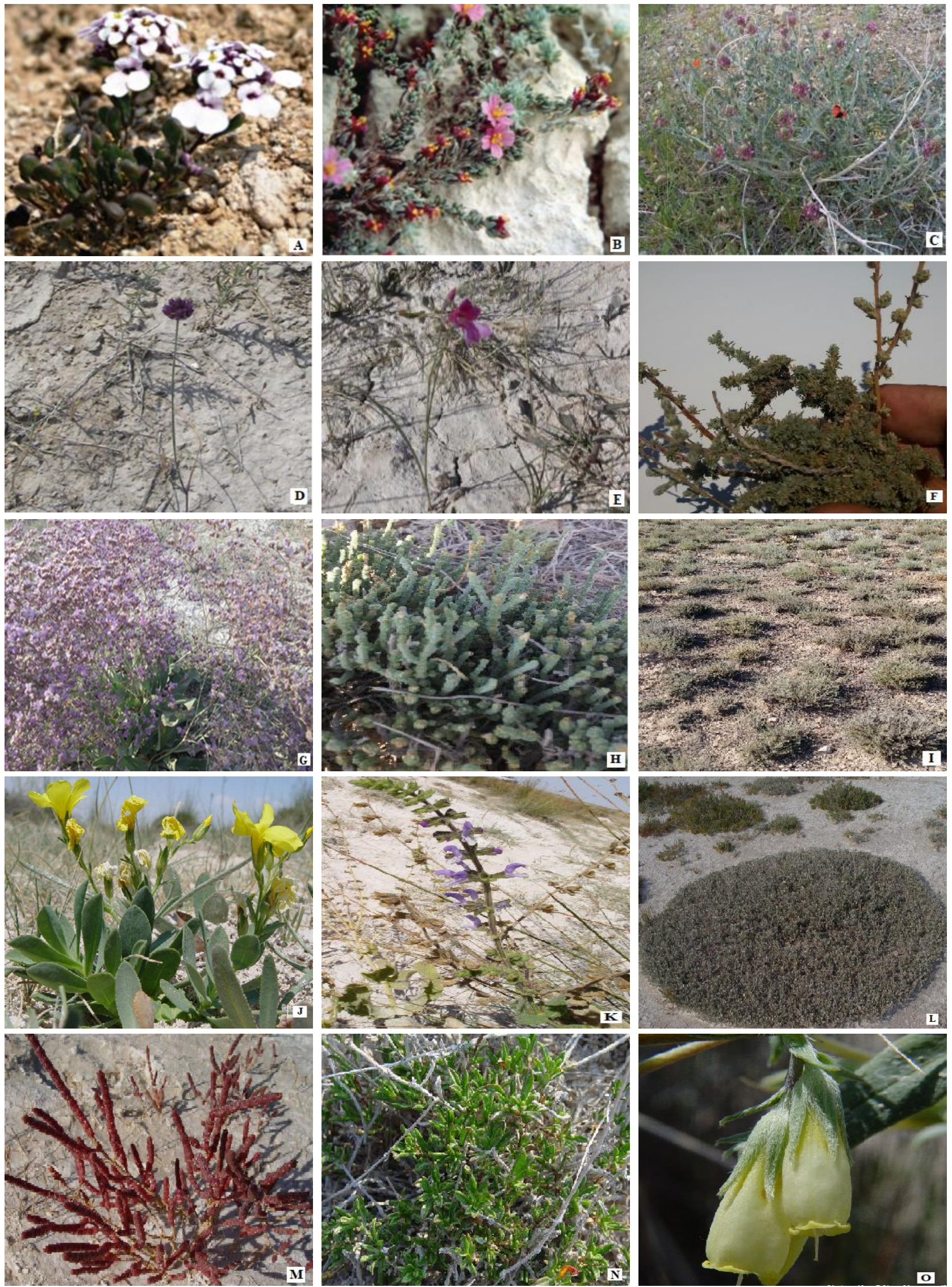

A. Iberis halophila, B. Frankenia salsuginea (Vural et al., 2012). C. Astragalus ovalis, D. Allium scabriflorum, E. Gladiolus halophilus (Furtana, 2010), F. Camphorosma monspeliaca, G. Limonium gmelinii. H. Halocnemum strabilaceum. I. Artemisia santonicum J. Linum ertugrulii, K. Salvia halophila, L. Halimione verrucifera, M. Microcnemum coralloides, N. Silene salsuginea (Photos of J,K,L,M,N: Prof. Dr. Kuddisi ERTUĞRUL), O. Onosma halophila (Photo: Prof. Dr. Osman TUGAY). 
Camphorosma species from the Chenopodiaceae family are preferred halophytes in landscape design, which can be grown in medium and very salty environments due to their high nutritional value and soil conservation from erosion (Huiskes, 1995). Halophytes are mainly suitable for restoration of disturbed landscapes, control of erosion and reduction of energy and water consumption (Cassaniti and Romano, 2011). Therefore, the prostrate species used as groundcover, like Halimione, Acantholimon, Halocnemum, Camphorosma and Frankenia seem to be of particular interest. In the soil analyzes made in August, taxons belonging to Halocnemum, Plantago and Frankenia genera prefer places where salt concentration is high (Tuğ, 2006). In terrestrial salt marshes, zonation varies from year to year, and the changes in species composition is due to changes in soil salinity (Ungar et al., 1979; Sözeri and Tipırdamaz, 2001). Although the soil parameters are effective on the distribution of $H$. strobilaceum, it can spread into the less salty steppes around Tuz Lake under low competition. EC, $\mathrm{Na}^{+}, \mathrm{SO}_{4}^{-}$and amount of total cations reflect soil salinity and very effective on plant growth and adaptation under saline conditions (Tuğ et al., 2008). The soil balance of $\mathrm{Ca}^{2+} / \mathrm{Mg}^{2+}, \mathrm{Ca}^{2+} / \mathrm{Na}^{+}$, and $\mathrm{K}^{+} / \mathrm{Na}^{+}$explain the edaphic ionic preferences of the species growing in dry salt marshes. On the other hand, maximum sodium adsorption ratio, maximum $\mathrm{Mg}^{2+}$ content, mean $\mathrm{Ca}^{2+}$ content, and mean $\mathrm{Ca}^{2+} / \mathrm{Mg}^{2+}$ ratio are the variables explaining the data set in the wet salt marshes which are the habitats of Juncus maritimus (Rogel et al., 2000).

The majority of the selected plants prefer salt concentrations of lower intensity. Some of the selected halophytes survive higher concentrations, however, they display characteristics which decrease their values as landscape plants. The use of halophytes in revegetation could be useful for biodiversity maintenance (Morales et al., 2001), saline soil desalinization, and for increasing crop yield on soils with marginal salinity (Albaho and Green, 2000).

\section{Conclusion}

The use of native halophytes in landscape design, along with the costs of water, maintenance, pesticides, fertilizer as well as the removal of salt-storing herbal components from habitat, will help to heal saline soils. Halophytes will be a sustainable alternative in arid and barren lands, which may be the result of possible global warming threats in the future, as they are well adapted to both wetlands and arid areas. These halophytes fulfill important qualities in landscape engineering, such as desalination, groundcover, erosion prevention or ornamental plant. Priority should be given to natural and local species that have adapted to the extreme conditions in the restoration of the semi-arid salty ecosystems. Despite growing in different geographies, foreign species successfully adapted to stress elements such as insufficient moisture and nutrients, high temperature and evaporation should not be ignored.

\section{References}

Akbaş F, Güvensen A. 2001. "Ege bölgesinin halofit vejetasyonunun Ekolojisi”, Köy Hizmetleri Genel Müdürlüğü APK Dairesi Başkanlığı Toprak ve $\mathrm{Su}$ Kaynakları Araştırma Şube Müdürlüğü, Toprak ve Su Kaynakları Araştırma Yıllığı, Ankara, 117: 297-310.

Abd El-Ghani MM. 2000. Vegetation composition of Egyptian inland saltmarshes. Bot Bull Acad Sinica, 41: 305-314.

Albaho MS, Green J. 2000. Suaeda salsa, a desalinating companion plant for greenhouse tomato. Horticultural Science, 35: 620-623.

Anonymous. 1992. Konya İli Arazi Varlığı. Tarım ve Köy İşleri Bakanlığı, Köy Hizmetleri Müdürlüğü Yayını, İl Rapor No:42, Ankara.

Aschenbach TA. 2006. Variation in growth rates under saline conditions of Pascopyrum smithii (Western Wheatgrass) and Distichlis spicata (Inland Saltgras) from different source populations in Kansas and Nebraska: Implications for the restoration of salt-affected plant communities. Restoration Ecology, 14: 21-27.

Breckle SW. 1983. Studies on halophytes from Iran and Afghanistan. II Ecology of Halophytes Along Salt Gradients, Royal Society of Edinburg, Proceedings Sec. B: Biological 89 B, 203-215.

Cassaniti C, Romano D. 2011. The use of halophytes for Mediterranean landscaping. The European Journal of Plant Science and Biotechnology, 5: 58-63.

Clark JR, Matheny NP. 1998. A model of urban forest sustainability: application to cities in the United States. Journal of Arboriculture, 24: 112-120.

Chapman VJ. 1974. Salt marshes and salt deserts of the world. In: Reimold, R.J. \& Queen, W.H. (eds), Ecology of Halophytes, pp. 3-19. Acad. Press Inc., New York \& London. A Subsidiary of Harcourt Brace Jovanovich Publishers.

Choukr-Allah R. 1991. The use of halophytes for the agricultural development of the southern part of Morocco. Plant Salinity Research New Challenges, IAV Hassan II, Agadir, Morocco: pp. 377-386.

Çakır YB, Özbucak T, Kutbay HG, Kılıç D, Bilgin A, Huseyinova R. 2010. Nitrogen and phosphorus resorption in a salt marsh in northern Turkey. Turkish Journal of Botany, 34: 311-322. doi:10.3906/bot-0906-64

Davis PH (ed.). 1965-1985. Flora of Turkey and the East Aegean Islands. Vols. 1-9. Edinburgh University Press, Edinburgh.

Deliboran A, Savran Ş. 2015. Toprak Tuzluluğu ve Tuzluluğa Bitkilerin Dayanım Mekanizmaları. Türk Bilimsel Derlemeler Dergisi, 8 (1): 57-61.

Deniz B, Şirin U. 2005. Samson Dağı dogal Bitki örtüsünün otsu karakterdeki bazı örneklerinden peyzaj mimarlig1 uygulamalarında yararlanma olanaklarının irdelenmesi. ADÜ Ziraat Fakültesi Dergisi, 2(2): 5-12.

Eroğlu E, Acar C, Demirel A. 2013. Yol şevlerindeki doğal yerörtücü bitkilerin peyzaj mimarlığında değerlendirilebilme olanakları: Sultanmurat-Uzungöl yol güzergahı örneği. V. Süs Bitkileri Kongresi Bildiriler Kitabı. 2: 126-134. 06-09 May 2013, Yalova.

Eyüpoğlu F. 1999. Türkiye Topraklarının verimlilik Durumu. Toprak ve Gübre Araştırma Enstitüsü Yayınları, Genel Yayın No: 220, Teknik Yayınlar No: T. 67, Ankara

Flowers TJ, Colmer TD. 2008. Salinity tolerance in halophytes. New Phytologist, 179: 945-963.

Flowers TJ, Troke PF, Yeo AR. 1977. Mechanism of salt tolerance in halophytes. Annual Review of Plant Physiology, 28: 89-121. 
Furtana GB. 2010. The ecophysiology of some endemic halophyte plants around the salt lake. Gazi University, Institute of Science and Technology. Ph. D. Thesis.

Güner A, AsIan S, Ekim T, Vural M, Babaç MT (edlr.). 2012. Türkiye Bitkileri Listesi (Damarlı Bitkiler). Nezahat Gökyiğit Botanik Bahçesi ve Flora Araştırmaları Derneği Yayını. istanbul.

Hamzaoğlu E, Aksoy A. 2009. Phytosociological studies on the halophytic communities of Central Anatolia. Ekoloji, 18(71): 1-14.

Hansen G, Alvarez E. 2010. Landscape design: Aesthetic characteristics of plants. ENH1172, IFAS Extension. University of Florida. http://edis.ifas.ufl.edu. (Accessed: 12.02.2018).

Huiskes AHL. 1995. Saline crops: A contribution to the diversification of the production of vegetable crops by research on the cultivation methods and selection of halophytes. Annual Report, 303p.

Jackson GB. 1914. Selecting Landscape Plants. Extension Service of Mississippi State University, cooperating with U.S. Department of Agriculture, Publication Number: P0666. http://extension.msstate.edu/sites/default/files/publications/p ublications/P0666.pdf (Accessed: 26.02.2018).

Koç N, Güneş G, 1998. Çatı Bahçelerinde Bitkisel Düzenleme Esasları, Mühendislik Bilimleri Dergisi. 4 (1-2): 625-633.

Korkut A, Kiper T, Topal TÜ. 2017. Kentsel peyzaj tasarımda ekolojik yaklaşımlar. Artium, 5(1): 14-26.

Martin T. 1986. "Finding a use for plants that Like Salt". Garden. Sept./Oct., 24-26.

Menemen Y, Hamzaoglu E. 2000. A new species of Dianthus (Caryophyllaceae) from Salt Lake, Central Anatolia, Turkey. Annales Botanici Fennici, 37: 285-287.

Morales MA, Torrecillas A, Rodríguez P, Alarcón JJ, SánchezBlanco MJ. 2001. Effects of salinity on growth and leaf water relations of two genotypes of Limonium sp. Acta Horticulturae, 559: 419-423.

O'leary JW. 1994. The Agricultural use of native plants on problem soils. Monographs on Theoretical and Applied Genetics, 21: 127-143.

Ellenberg H, Mueller-Dombois D. 1967. A key to Raunkiaer plant life forms with revised subdivisions. Berichte des Geobotanischen Institutes der E.T.H. Stiftung Rübel Zürich 37: 56-73.
Robert FB, Richard LH, Susan JM. 2007. Landscape Architects' use of native plants in the Southeastern United States. HortTechnology, 17(1): 78-81.

Rogel JA, Ariza FA, Silla RO. 2000. Soil salinity and moisture gradients and plant zonation in Mediterranean salt marshes of Southeast Spain. Wetlands, 20: 357-372.

Sánchez-Blanco MJ, Rodríguez P, Morales MA, Ortuño MF, Torrecillas A. 2002. Comparative growth and water relations of Cistus albidus and Cistus monspeliensis plants during water deficit conditions and recovery. Plant Science, 162: 107-113.

Sözeri S, Tıpırdamaz R. 2001. Seyfe gölü (Kırşehir) çevresinin doğal mera alanlarındaki halofit (tuzcul) bitkileri ve bunlardan yararlanma olanakları. Türk Herboloji Dergisi, 4(2): 11-35.

Tanay Y, Güney MA, Türel HS, Kılıçaslan Ç. 2006. Bitkisel Tasarım. Ders Kitabı. ISBN:9944-5419-0-7. İzmir.

Thomas GS. 2004. The Rock Garden and It's Plants, Sagapress Inc., Timber Press Inc., Portland, Oregon.

Tugay O, Bağcı Y, Uysal T. 2010. Linum ertugrulii (Linaceae), a new species from Central Anatolia, Turkey. Annales Botanici Fennici, 47(2): 135-138.

Tuğ GN. 2006. "Determination of the factors effective on zonation of halophytic vegetation of salt lake, inner Anatolia, Turkey. Ankara University Graduate School of Natural and Applied Sciences, Department of Biology. Ph. D. Thesis.

Tuğ GN, Yaprak AE, Ketenoğlu O. 2008. Soil determinants for distribution of Halocnemum strobilaceum Bieb. (Chenopodiaceae) around Lake Tuz, Turkey. Pakistan Journal of Biological Sciences, 11(4): 565-570.

Ungar IA, Benner DK, McGraw DC. 1979. The distribution and growth of Salicornia europaea on an inland salt pan. Ecology 60: 329-336

Vural M, Duman H, Aytaç Z, Adıgüzel N. 2012. A new genus and three new species from Central Anatolia, Turkey Turkish Journal of Botany, 36: 427-433. doi:10.3906/bot1105-16

Yaprak AE, Tug GN. 2006. Halophytic endemics of Turkey. Plant, fungal and habitat diversity investigation and conservation. Proceedings of IV BBC - Sofia.

Zahran M. 1982. Vegetation types of Saudi Arabia. Jeddah: King Abdel Aziz University Press

Zia S, Egan TP, Khan MA. 2008. Growth and selective ion transport of Limonium stocksii Plumbaginacea under saline conditions. Pakistan Journal of Botany, 40: 697-709. 